\title{
THROUGHPUT AND NOISE OF THE ALADDIN INFRARED BEAMLINE*
}

\author{
R. A. Bosch, ${ }^{\#}$ R. L. Julian, R. W. C. Hansen, M. A. Green, K. J. Kleman and K. D. Jacobs, \\ Synchrotron Radiation Center, University of Wisconsin-Madison, \\ 3731 Schneider Dr., Stoughton, WI 53589, USA
}

\begin{abstract}
The infrared beamline at the Aladdin electron storage ring utilizes edge radiation for spectromicroscopy. For wavelengths of $0.8-16 \mu \mathrm{m}$, computations indicate that $\sim 20 \%$ of the collected radiation is transported through the microscope. Transverse oscillations of the electron beam cause oscillations in microscope throughput that account for about one-third of the beamline's zero-burst noise.
\end{abstract}

\section{BEAMLINE THROUGHPUT}

The Aladdin infrared (IR) microspectroscopy beamline [1] transmits and detects radiation at wavelengths of 0.8 $16 \mu \mathrm{m}$. The lower end of this range is determined by transmission of a $\mathrm{KBr}$ beam splitter substrate, while the upper end results from the detector response. To study its performance with our low-emittance electron beam [2], we model our beamline with the SRW code [3]. We neglect flat mirrors and treat focusing mirrors as ideal thin lenses, as shown in Fig. 1. The source is edge radiation from a $200 \mathrm{~mA}, 800 \mathrm{MeV}$ electron beam traversing a straight section of length $L=3 \mathrm{~m}$, terminated by $1.28 \mathrm{~T}$ bending magnets. The bending magnet fringe field length is $108 \mathrm{~mm}$, while the entrance aperture is at distance $R=$ $1.5 \mathrm{~m}$ from the edge-radiation source at the downstream end of the straight section. For our beamline, a $1-\mu \mathrm{m}$ source translation causes a $3.1-\mu \mathrm{m}$ translation and $6.6-\mu \mathrm{rad}$ deflection of the photon beam at the microscope entrance.

In transmission mode, the microscope is expected to admit and focus an annulus of collimated radiation with outer diameter of $7 \mathrm{~mm}$ and inner diameter of $3.5 \mathrm{~mm}$. In our model, we assume that all radiation admitted by the microscope is transmitted through it.

The SRW code computes emittance effects for an electron beam that undergoes no focusing. Since the Aladdin 3-m straight section contains two quadrupole magnets near its midpoint, an approximate description is necessary. One approach is to consider an electron beam with constant size $\left(\sigma_{x}, \sigma_{y}\right)=(200 \mu \mathrm{m}, 100 \mu \mathrm{m})$. This method approximately describes the low-emittance beam size throughout the straight section, while neglecting its divergence, which is smaller than the IR-radiation opening angle. Another approach is to specify the lattice functions at the downstream end of the straight section to model the beam size and divergence downstream of the quadrupoles, while misrepresenting the upstream half of the straight section. We obtained similar results with both approaches, and show modeling of a constant beam size.

\footnotetext{
* Work supported by NSF grant DMR-0084402.

\# bosch@src.wisc.edu
}

Figure 2 displays flux computations at wavelengths $\lambda$ of 1 and $10 \mu \mathrm{m}$. At the entrance aperture, a flux of $2-3 \times 10^{13}$ photons $/ \mathrm{s}-0.1 \%$ bandwidth is obtained for wavelengths of $0.8-16 \mu \mathrm{m}$. Wavelengths exceeding $\sim 1 \mu \mathrm{m}$ form an asymmetric hollow ring with radius of $\sim[\lambda R(R+L) / L]^{1 / 2}$. At the microscope entrance, the collimated photon beam forms an asymmetric hollow ring with radius of $\sim(\lambda D)^{1 / 2}$, where $D=6 \mathrm{~m}$ is the length of the collimated photon beam. These radii equal the diffraction-limited sizes of near-field edge radiation and a collimated photon beam.

In contrast, SRW modeling of a source of ordinary synchrotron radiation (located $1.5 \mathrm{~m}$ from the entrance aperture) shows radiation bands at the top and bottom of the entrance aperture, and a $30 \mathrm{~mm} \times 20 \mathrm{~mm}$ rectangle of collimated radiation at the microscope. While radiation from an edge-radiation source is transported to the microscope quite effectively at wavelengths of $0.8-16$ $\mu \mathrm{m}$, the beamline greatly overfills the microscope when used with ordinary synchrotron radiation.

At a representative wavelength of $3 \mu \mathrm{m}$, edge radiation provides $77 \%$ more flux into the entrance aperture than synchrotron radiation. When best aligned for $\lambda=3 \mu \mathrm{m}$, the microscope admits $20 \%$ of the edge radiation incident upon the entrance aperture, but only $5 \%$ of the ordinary synchrotron radiation. For our beamline, edge radiation is computed to increase the $3-\mu \mathrm{m}$ flux through the microscope by a factor of seven.

By computing the flux distribution for several wavelengths, the distribution of power in the wavelength range of $0.8-16 \mu \mathrm{m}$ was computed at the microscope entrance plane. Figure 3(a) shows the power admitted by the microscope versus photon beam position (i.e., optical axis position with respect to the microscope). In the wavelength range of $0.8-16 \mu \mathrm{m}$, the power collected by the entrance aperture is $5.7 \mathrm{~mW}$. When best aligned, the microscope admits $18 \%$ of this collected power. In

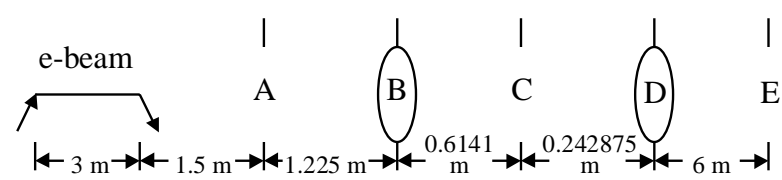

Figure 1. The IR source is a $3-\mathrm{m}$ straight section of the electron storage ring. Beamline components are:

A. Entrance aperture, $30.5 \mathrm{~mm}$ hor. $\times 22.3 \mathrm{~mm}$ vert.

B. Focusing lens, focal length $=459 \mathrm{~mm}$, diameter $=$ $76.2 \mathrm{~mm}$.

C. Diamond window, diameter $=10 \mathrm{~mm}$.

D. Collimating lens, focal length $=305 \mathrm{~mm}$, diameter $=50.8 \mathrm{~mm}$.

E. Entrance of the IR microscope. 
contrast, the microscope admits only $\sim 5 \%$ of the collected power in this wavelength range from a source of ordinary synchrotron radiation.

For a given photon beam position, dividing the slope in Fig. 3(a) by the admitted power gives the relative sensitivity to a horizontal change in photon-beam position. For photon-beam positions of $-0.7,-1.4$, or $-3.6 \mathrm{~mm}$, the slope is zero, giving "optimal" horizontal alignment in which the admitted power is insensitive to a small horizontal motion of the photon beam.
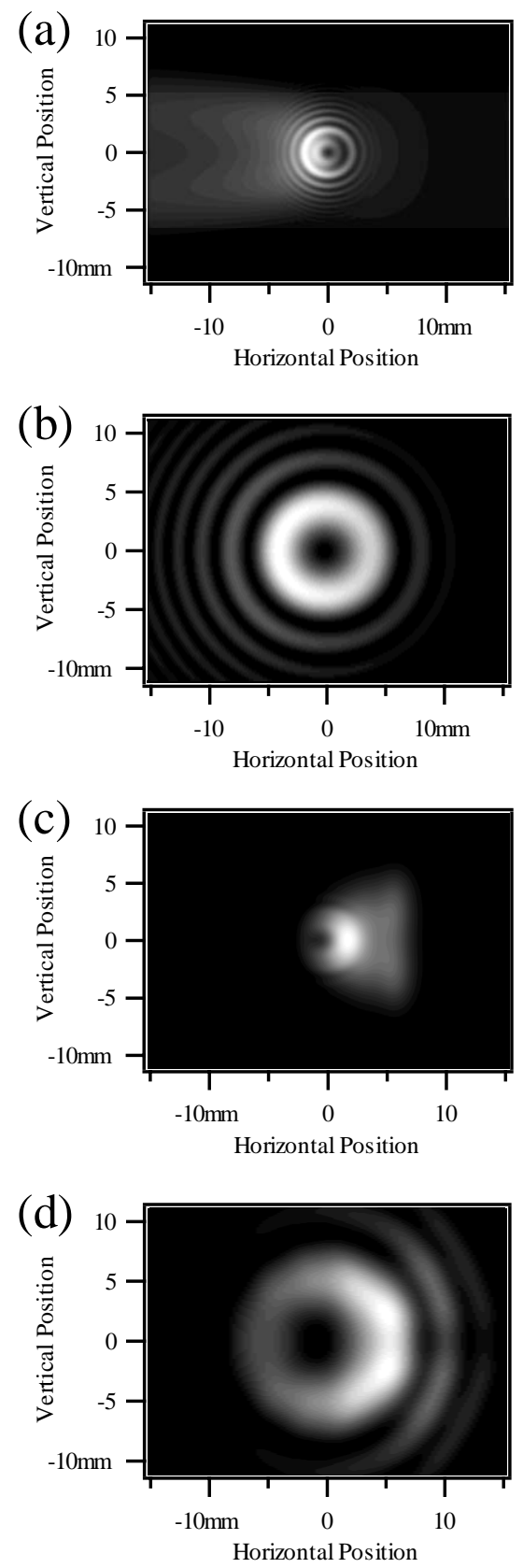

Figure 2. Infrared edge radiation modeled by SRW.
(a) $\lambda=1 \mu \mathrm{m}$ at the entrance aperture.
(b) $\lambda=10 \mu \mathrm{m}$ at the entrance aperture.
(c) $\lambda=1 \mu \mathrm{m}$ at the entrance of the microscope.
(d) $\lambda=10 \mu \mathrm{m}$ at the entrance of the microscope.

When the microscope is operated in reflection mode, it is expected to admit radiation in the upper half of the annulus admitted by transmission mode. For an optimal horizontal photon beam position of $-3.6 \mathrm{~mm}$, Fig. 3(b) shows the computed power in transmission and reflection modes. With optimal vertical alignment for transmission mode, the reflection-mode sensitivity to a vertical photon beam motion is $0.013 \% / \mu \mathrm{m}$. When using a single alignment for both modes, one mode is computed to have a vertical sensitivity of $0.01-0.02 \% / \mu \mathrm{m}$.

Experimentally, we maximized the zero-burst signal (which measures power through the microscope) by translating the electron beam from its standard position by -540 um horizontally and $-190 \mu \mathrm{m}$ vertically. The associated change in photon beam position is $-1.7 \mathrm{~mm}$ horizontal and $-0.6 \mathrm{~mm}$ vertical. The zero burst increased $15 \%$, while the flux increased by $>8 \%$ for wavelengths of $0.8-16 \mu \mathrm{m}$. Figure 4 shows the zero-burst signal as the electron beam was translated. The difference between Figs. 3 and 4 may result from the wavelength dependence of optical elements and detector, uncertainty in the photon beam position, lens aberrations, and additional apertures.

\section{IR BEAMLINE NOISE}

The Aladdin IR beamline is adversely affected by motion of the electron beam at audio frequencies [4]. The

(a)
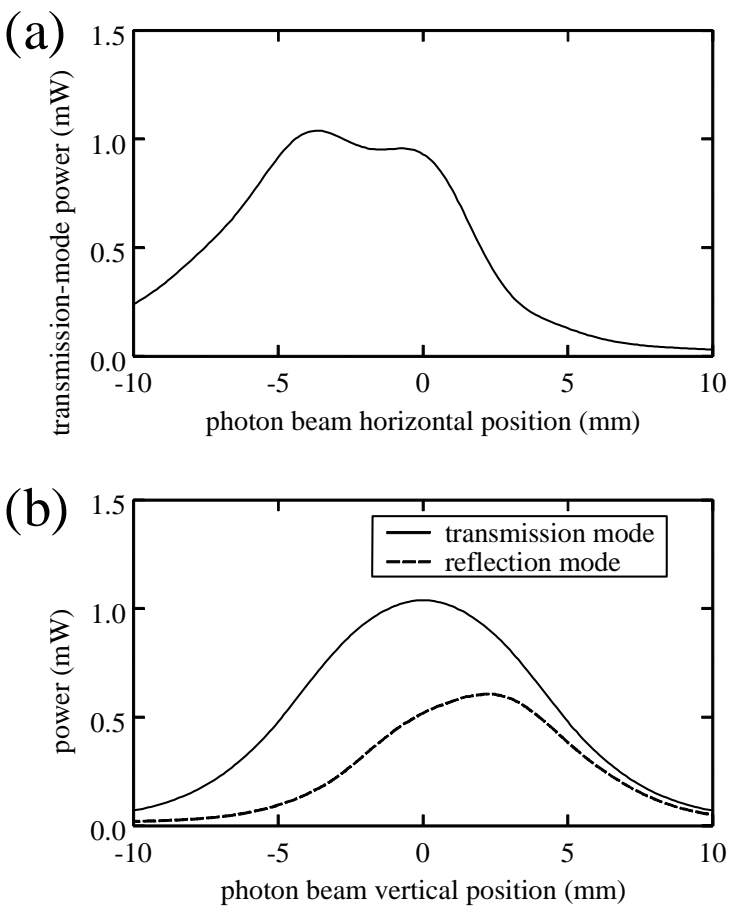

Figure 3. Computation of power admitted by the microscope for wavelengths of $0.8-16 \mu \mathrm{m}$.

(a) Transmission-mode power versus horizontal photon beam position, where the vertical photon beam position equals zero.

(b) Transmission-mode and reflection-mode power versus vertical photon beam position, where the horizontal photon beam position equals $-3.6 \mathrm{~mm}$. 
zero-burst signal variation is in the range of $0.15-0.25 \%$ rms. A portion of this variation may be caused by transverse motion of the electron beam.

The transverse electron motion is dominated by a $60-\mathrm{Hz}$ oscillation in which the horizontal and vertical positions vary by $\sim 1 \mu \mathrm{m} \mathrm{rms}$; the oscillation of the beam's direction of propagation is unknown. The motion is comparable in horizontal and vertical directions, in non-equivalent bending magnets, and with high- and low-emittance beams. This suggests that a large portion of the $60-\mathrm{Hz}$ oscillation is caused by the electromagnetic field of ripple current penetrating the vacuum chamber [5].

For the standard electron beam position, the horizontal sensitivity to photon beam motion from Fig. 4 is $\sim 0.01 \% / \mu \mathrm{m}$ and the vertical sensitivity is $\sim 0.02 \% / \mu \mathrm{m}$. Electron-beam oscillations of $\sim 1 \mu \mathrm{m}$ rms are expected to cause photon-beam oscillations of $\sim 3.1 \mu \mathrm{m} \mathrm{rms}$, causing a zero-burst variation of $\sim 0.06 \% \mathrm{rms}$, about one-third of its measured value. Translating the electron beam to maximize the zero-burst signal (giving optimal alignment) reduced the zero-burst variation by $23 \%$.

A subsequent realignment of the microscope and interferometer increased the zero-burst signal in transmission mode by $20 \%$ while achieving a reflectionmode signal equal to one-half of that in transmission mode. With this alignment, the transmission mode signal was $20 \%$ below the maximum achievable. According to Fig. 4(b), the transmission-mode sensitivity to a vertical photon beam translation is $\sim 0.03 \% / \mu \mathrm{m}$ when the zero
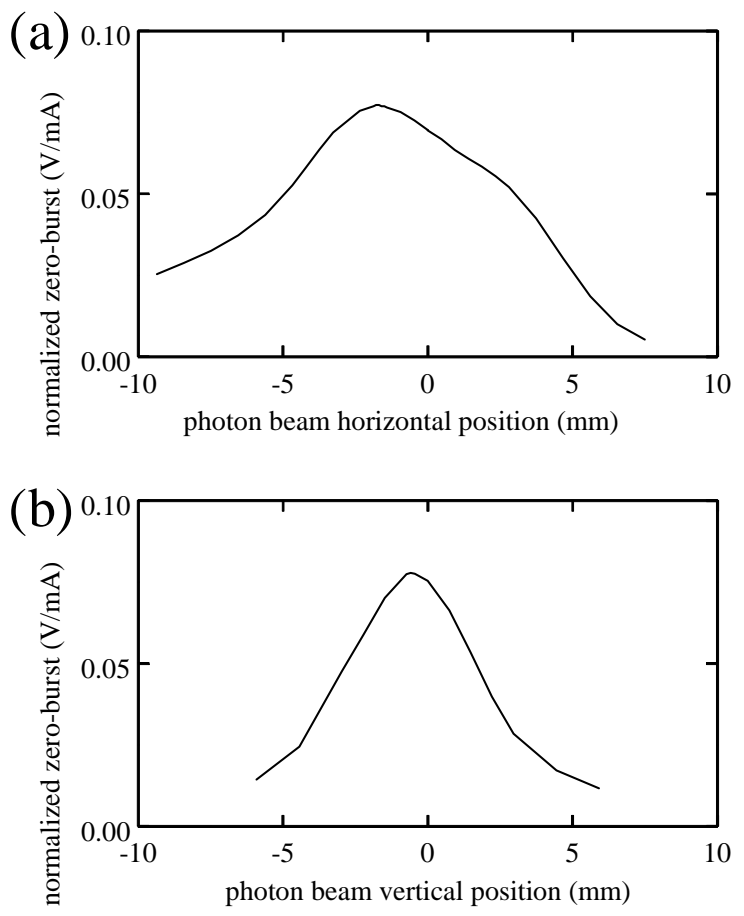

Figure 4. Zero-burst voltage in transmission mode as the photon beam is scanned (a) horizontally and (b) vertically through the position of maximum zero burst. For the standard electron beam position, the photon beam is at $(0,0)$. burst is $20 \%$ below maximum. The expected zero-burst variation caused by transverse motion of the electron beam is $\sim 0.09 \% \mathrm{rms}$, about $50 \%$ larger than before the realignment. Consistent with this expectation, the realignment increased the zero-burst variation by $21 \%$ in transmission mode. This zero-burst variation is $60 \%$ larger than that observed when the electron beam was translated to achieve optimal transmission-mode alignment, suggesting that about one-third of the zeroburst noise arises from transverse electron-beam motion. Oscillations of the electron-beam size and longitudinal bunch positions, as well as detector background, may also contribute to the zero-burst noise.

At frequencies $>100 \mathrm{~Hz}$, beam motion is primarily driven by radiofrequency (RF) system noise [5]. For these frequencies, the noise spectrum of a detector on the IR beamline is similar to that of the electron beam's longitudinal phase, peaking at the $3-4 \mathrm{kHz}$ frequencies of Robinson modes [6]. We have recently installed a crystal master oscillator from Wenzel Associates and improved the common-mode rejection and high frequency filtering of the RF system. This has reduced RF noise by 20-30 $\mathrm{dB}$. Noise at frequencies around $3 \mathrm{kHz}$ has been reduced an additional $20 \mathrm{~dB}$ by feedback that damps oscillations of the bunch centroids [6]. These reductions of RF noise greatly improve the IR beamline performance, but have only a small effect on the zero-burst signal variation. This indicates the limitation in using the zero-burst signal variation to characterize the beamline's performance.

\section{SUMMARY}

For wavelengths of $0.8-16 \mu \mathrm{m}$, SRW computations indicate that $\sim 20 \%$ of the collected edge radiation is transported through the microscope. Experiments and modeling suggest that about one-third of the IR zero-burst noise in transmission mode is caused by $60-\mathrm{Hz}$ transverse oscillations of the electron beam. Since a single microscope alignment is used for both transmission and reflection modes, this noise was not reduced by realignment. Modifications to the RF system have reduced noise in the $1-6 \mathrm{kHz}$ range by $20-40 \mathrm{~dB}$, greatly improving IR beamline performance.

\section{REFERENCES}

[1] T. E. May, R. A. Bosch and R. L. Julian, in Proc. 1999 PAC (IEEE, Piscataway, NJ, 1999), p. 2394.

[2] J. J. Bisognano, R. A. Bosch, D. E. Eisert, M. A. Green, K. J. Kleman and W. S. Trzeciak, in Proc. 2001 PAC (IEEE, Piscataway, NJ, 2001), p. 2671.

[3] O. Chubar and P. Elleaume, in Proc. Sixth European PAC (IOP, Bristol, 1998), p. 1177.

[4] R. A. Bosch and R. L. Julian, Rev. Sci. Instrum. 73, 1420 (2002).

[5] R. Biscardi, G. Ramirez, G. P. Williams and C. Zimba, Rev. Sci. Instrum. 66, 1856 (1995).

[6] R. A. Bosch, K. J. Kleman and J. J. Bisognano, "Robinson modes at Aladdin," these proceedings. 